\title{
Outcome Evaluation of Early Implementation of Option B+ in Cameroon: A Prospective Cohort Observational Survey in the Northwest and Southwest Regions
}

\author{
Pius Tih Muffih1, Edouard Katayi Tshimwanga1*, Andrew Abutu², Lem Edith Abongwa1, \\ Jacques Chirac Awa1, Pascal Nji Atanga1, Felix Desembuin', Eveline Mboh Khan', \\ Kuni Esther Bonje1, Nshom Emmanuel1, Ebeny Francois Temgbait Chimoun1, \\ Thomas Welty', Gladys Tayong Fosah ${ }^{3}$, Jennifer Lim ${ }^{4,5}$, Dana Duncan², Leah Petit', \\ Gilbert Tene $^{5,7}$, Jembia Mosoko5, Omotayo Bolu ${ }^{5}$

\begin{abstract}
${ }^{1}$ AIDS Care and Prevention Program, Cameroon Baptist Convention Health Services, Bamenda, Cameroon
${ }^{2}$ United States Centers for Disease Control and Prevention (CDC), Atlanta, USA

${ }^{3}$ Ministry of Public Health, National AIDs Control Committee, Northwest Region Technical Group, Bamenda, Cameroon

${ }^{4}$ Association of Schools and Programs of Public Health/Allan Rosenfield Global Health Fellowship, Washington DC, USA

${ }^{5}$ United States Centers for Disease Control and Prevention (CDC), Yaoundé, Cameroon

${ }^{6}$ Elizabeth Glazer Pediatric AIDS Foundation (EGPAF), Washington DC, USA

${ }^{7}$ QED Group, LLC, Washington DC, USA
\end{abstract} \\ Email: *edouarddr@yahoo.fr, fchimoun@gmail.com
}

How to cite this paper: Muffih, P.T., Tshimwanga, E.K., Abutu, A., Abongwa, L.E., Awa, J.C., Atanga, P.N., Desembuin, F., Khan, E.M., Bonje, K.E., Emmanuel, N., Chimoun, E.F.T., Welty, T., Fosah, G.T., Lim, J., Duncan, D., Petit, L., Tene, G., Mosoko, J. and Bolu, O. (2018) Outcome Evaluation of Early Implementation of Option B+ in Cameroon: A Prospective Cohort Observational Survey in the Northwest and Southwest Regions. World Journal of AIDS, 8, 90-104.

https://doi.org/10.4236/wja.2018.83008

Received: May 30, 2018

Accepted: September 18, 2018

Published: September 21, 2018

\begin{abstract}
Background: Mother to child transmission (MTCT) of HIV constitutes a major source of new pediatric infections in Cameroon. Objective: The aim of this implementation research was to assess outcomes and effectiveness of providing life-long antiretroviral therapy (ART) for HIV-positive pregnant and breastfeeding women (Option B+). Methods: From October 2013 to July 2014, HIV-positive pregnant and breastfeeding women, not on antiretroviral (ARV) prophylaxis and ART, were recruited from 22 purposefully selected health facilities in the Northwest and Southwest regions for a prospective, observational cohort evaluation. Option $\mathrm{B}+$ was offered to participants and outcome indicators were measured. Results: Out of 680 women eligible for this assessment, 669 (98\%) were initiated on Option B+. Retention-in-care was $90 \%$ (95\% CI, 87.85 - 92.61) and 79\% (95\% CI, 75.20 - 81.88), and loss to follow up (LTFU) was 7\% (95\% CI: 4.95 - 8.90) and 15\% (95\% CI: 12.06 17.56) at 6 and 12 months respectively. Maternal mortality at 12 months after ART initiation was 2\% (13). As of March 2015, 538 HIV exposed infants (HEIs) were enrolled and received postpartum nevirapine prophylaxis within
\end{abstract}


Copyright $\odot 2018$ by authors and Scientific Research Publishing Inc. This work is licensed under the Creative Commons Attribution International License (CC BY 4.0).

http://creativecommons.org/licenses/by/4.0/

(c) (i) Open Access
72 hours of birth and $84 \%$ (454) were on cotrimoxazole at 6 to 8 weeks. By 8 weeks of age, 498 (93\%) infants had HIV DNA PCR test with 486 (97.6\%) negative and $12(2.4 \%)$ positive. Conclusion: In Cameroon, successful implementation of Option B+ increased retention-in-care to $79 \%$ at one year for pregnant and breastfeeding women and reduced MTCT rate below 5\% for HEIs at 8 weeks of age. Long term retention, maternal and infant mortality and final MTCT rate after cessation of breastfeeding require further evaluation.

\section{Keywords}

Option B+, Implementation Research, Prevention of Mother to Child HIV Transmission, HIV Exposed Infants

\section{Introduction}

With a population of 23.3 million and a national HIV prevalence of $4.5 \%$, Cameroon is one of the countries most affected by the human immunodeficiency virus and acquired immune deficiency syndrome (HIV/AIDS) epidemic in West and Central Africa [1] [2]. Accessibility of Prevention of Mother-to-Child Transmission (PMTCT) of HIV services remains low in the country. National data from 2011 showed that while $81 \%$ of pregnant women seen in health facilities received HIV testing during antenatal care (ANC), labor and delivery (L \& D), and the postpartum period combined (8.4\% tested HIV-positive), only $67 \%$ of these HIV-positive women received antiretroviral therapy (ART) for PMTCT [3] [4]. Although the Cameroon HIV guidelines in 2011 specified that all HIV-positive exposed infants (HEIs) were eligible for immediate ART, at the time this implementation research was initiated in 2013 , only $25 \%$ of HEIs were provided antiretroviral (ARV) prophylaxis at birth, 9.3\% had an HIV deoxyribonucleic acid polymerase chain reaction (DNA PCR) test at 8 weeks of age (7.1\% infants testing HIV-positive), and approximately $3 \%$ of those HIV infected were initiated on lifelong ART [4]. Consequently, HIV-related deaths have contributed to a decrease in life expectancy in Cameroon from 54 years in 1990 to 51.5 years in 2010 , with $10.1 \%$ of maternal deaths estimated to be HIV-related [5] [6].

In support of the national HIV/AIDS program in Cameroon, the Cameroon Baptist Convention Health Services (CBCHS), in collaboration with the Elizabeth Glaser Pediatric AIDS Foundation (EGPAF), the Centers for Disease Control and Prevention (CDC), and the Cameroon Ministry of Public Health $(\mathrm{MOPH})$, initiated scaling up implementation of HIV testing services (HTS), care and treatment, and PMTCT services with the aim to reduce HIV burden among these vulnerable populations [7]. The PMTCT program has evolved from the use of single-dose nevirapine (sdNVP) in 2000 to implementation of the World Health Organization (WHO) Option A regimen for pregnant and breast- 
feeding women in 2010 [7] [8]. For HIV-infected pregnant women who were not eligible for lifelong ART based on CD4 count or clinical staging, Option A consisted of antepartum twice-daily zidovudine (ZDV) to HIV-infected pregnant women starting at 14 weeks of gestation and continued throughout pregnancy. At the onset of labor, sdNVP was administered with the initiation of twice daily ZDV and lamivudine (3TC) for seven days postpartum. HIV-exposed infant (HEI) prophylaxis involved neviparine (NVP) syrup from birth until one week after cessation of breastfeeding or for six weeks postpartum if not breastfeeding [9] [10]. In 2010, the government of Cameroon developed a national plan for the elimination of mother-to-child transmission of HIV (eMTCT) to align with the global eMTCT plan to reduce new pediatric HIV infections and decrease the MTCT rate to less than $5 \%$ by 2015 [11] [12].

In 2012, Cameroon's MOPH decided to implement Option B+ (life-long ART for pregnant and breastfeeding women regardless of $\mathrm{CD} 4$ count) nationwide using a phased approach to reach these goals [11]. With funding from the U.S. President's Emergency Plan For AIDS Relief (PEPFAR) through CDC, CBCHS piloted a rollout of PMTCT Option B+ in the Northwest and Southwest regions of the country. The aim of this study was to evaluate outcomes and effectiveness of PMTCT Option B+ service at 12 months postpartum in order to facilitate national scale-up by the MOPH. A second evaluation will describe 24 months postpartum outcomes.

\section{Materials and Methods}

\subsection{Study Design, Settings and Population}

A prospective cohort of mother-infant pairs were enrolled from maternal and child health $(\mathrm{MCH})$ services across 22 health facilities in the Northwest (Bamenda) and Southwest (Kumba) regions of Cameroon and followed monthly until 12 months after Option B+ initiation. The 22 health care facilities, 12 in Bamenda and 10 in Kumba, were purposely selected. Participants enrolled for the evaluation, from October 2013 to July 2014, were newly identified HIV positive pregnant or postpartum women in ANC, L \& D, and postpartum care who were not on ARV prophylaxis or antiretroviral therapy (ART). HIV positive breastfeeding women whose infants were greater than 18 months of age were excluded. As per Option B+ recommendations, clients were initiated on ART immediately after HIV diagnosis, and blood was drawn for baseline laboratory tests of CD4 cell count, full blood count, liver function test, serum creatinine, and fasting blood sugar. None of these tests delayed ART initiation. All costs associated with laboratory tests were absorbed by the project and study participants did not pay for any testing. All women who only accepted Option B received ongoing counseling throughout this period to continue ART for life.

\subsection{Sample Size Estimation}

Given the number of project sites with the expected population of HIV-positive 
women attending ANC services in the 10 months enrolment period, facility-specific sampling targets were determined by sampling proportionally to three patient volume categories (low, medium, and high) based on ANC attendance, PMTCT services utilization rate, and geographical accessibility. Effort was made to enrol participants from all volume sites resulting in the enrolment of 100 mother-infant pairs from low and medium volume sites, and 400 from high volume sites.

\subsection{Option B+ Implementation Support}

Task shifting with nurses and midwives, a defaulter tracking system and a laboratory sample transport system were implemented in all 22 study sites. $\mathrm{CBCHS}$ developed a training manual adapted from materials used in Option B+ implementation in Malawi to train physicians, nurses, and midwives. Eleven ART Clinic physicians, selected from the pilot districts, were trained on patient management in the context of Option B+. These physicians trained 109 nurses and midwives from study sites and were available for consultation or referral as needed. Twenty-nine peer educators were also trained to provide additional counseling, home visits, and community sensitization. Each of them had access to a mobile phone and transportation costs to facilitate home visit or phone contact. Bikers For Health (BFH) were hired to ensure timely transportation of maternal laboratory samples, infant dried blood spots (DBS), Early Infant Diagnosis (EID) commodities and HIV test kits. Short Message Service (SMS) technology was used in addition to $\mathrm{BFH}$ to accelerate results return from the reference laboratory.

\subsection{Data Collection and Analysis}

Nursing and midwifery staff in each health facility filled out PMTCT longitudinal maternal ART and HEIs registers following each visit. The registers were reviewed monthly by monitoring and evaluation officers who extracted data into an Epi Info database. Data were cleaned and analyzed using Epi Info version 3.5.4 and Stata/SE-13 software. Data analysis was performed on a monthly basis from October 2013 to October 2015, and results were reviewed during coordination meetings by the project implementation team to identify challenges and improve implementation.

\subsection{Outcomes and Definitions}

Outcomes of interest among pregnant and breastfeeding women initiating Option B+ were ART uptake, ART retention, stoppage of ART, loss to follow-up (LTFU), and mortality. For HEIs, the study assessed NVP and cotrimoxazole uptake, HIV status at 6 to 8 weeks, LTFU, and mortality. For each cohort, LTFU was defined as the proportion of patients who missed their ART pick up appointment for 90 consecutive days or more at 6 and 12 months after enrollment. Maternal ART retention was defined as: the proportion of HIV-positive pregnant 
and breastfeeding women on ART after initiating out of the total number of women who initiated ART and should have completed 6 and 12 months respectively. Thus, clients considered not retained or LTFU at 6 months could re-engage and be considered retained at the 12 months' time point. Clients who transferred out of the facilities were excluded from the retention analysis. Transferred out was defined as women who were referred and/or transferred to other facilities due to either miscarriage, stillbirth, infant death, or relocation, or an HIV DNA PCR positive infant. HIV infected infants were transferred to ART clinics for ART initiation but were also included in the MTCT rate computation for this evaluation. The ART retention denominator was the number of clients on ART excluding the number who transferred out for each cohort at 6 and 12 months.

\subsection{Ethical Considerations}

This study was approved as a public health program evaluation by the CBCHS Institutional Review Board in August 2013 and the CDC in July 2013. All participants were counseled in line with national protocols and were asked to give verbal consent before enrollment in the evaluation.

\section{Results}

Figure 1 shows the flow chart of participants enrolled in the study. Of 11,313 pregnant or breastfeeding women who attended maternal and child health $(\mathrm{MCH})$ services in the 22 study facilities, 680 (6\%) were HIV-positive and not on ART or ARV prophylaxis. Of these 680 eligible participants, 669 (98\%) initiated Option $\mathrm{B}+$; this included three clients who initially accepted ART only during pregnancy but later changed to lifelong ART during the course of the pregnancy. All 669 participants who initiated Option B+ were enrolled in the study.

\subsection{Maternal Demographic Characteristics}

The median age at enrollment was 27 years (IQR: 24 - 31 years). Of the 669 participants, $522(78 \%)$ were either married or cohabiting, 608 (91\%) owned a phone; 362 (54\%) preferred to be contacted by phone, and 270 (40\%) preferred to be contacted by phone or home visit. Of the 669 participants, a majority (58.7\%) spent less than 200 CFA (less than \$0.5 USD) to reach the health facility; 431 (64\%) lived in Bamenda and 238 (36\%) in Kumba. Six hundred and twenty-six (94\%) enrolled in the study during pregnancy, and 454 (68\%) were newly-diagnosed with HIV at their first presentation to $\mathrm{MCH}$ services. The median baseline CD4 count was 364 cells/mm³ (IQR: 225 - 496) (Table 1).

\subsection{ART, Retention, and Mortality among Pregnant and Breastfeeding Women}

Of the 669 participants, $43(6 \%)$ and $64(10 \%)$ clients were transferred out during the $0-6$ and $0-12$ month maternal cohort. Thus, 626 and 605 pregnant 


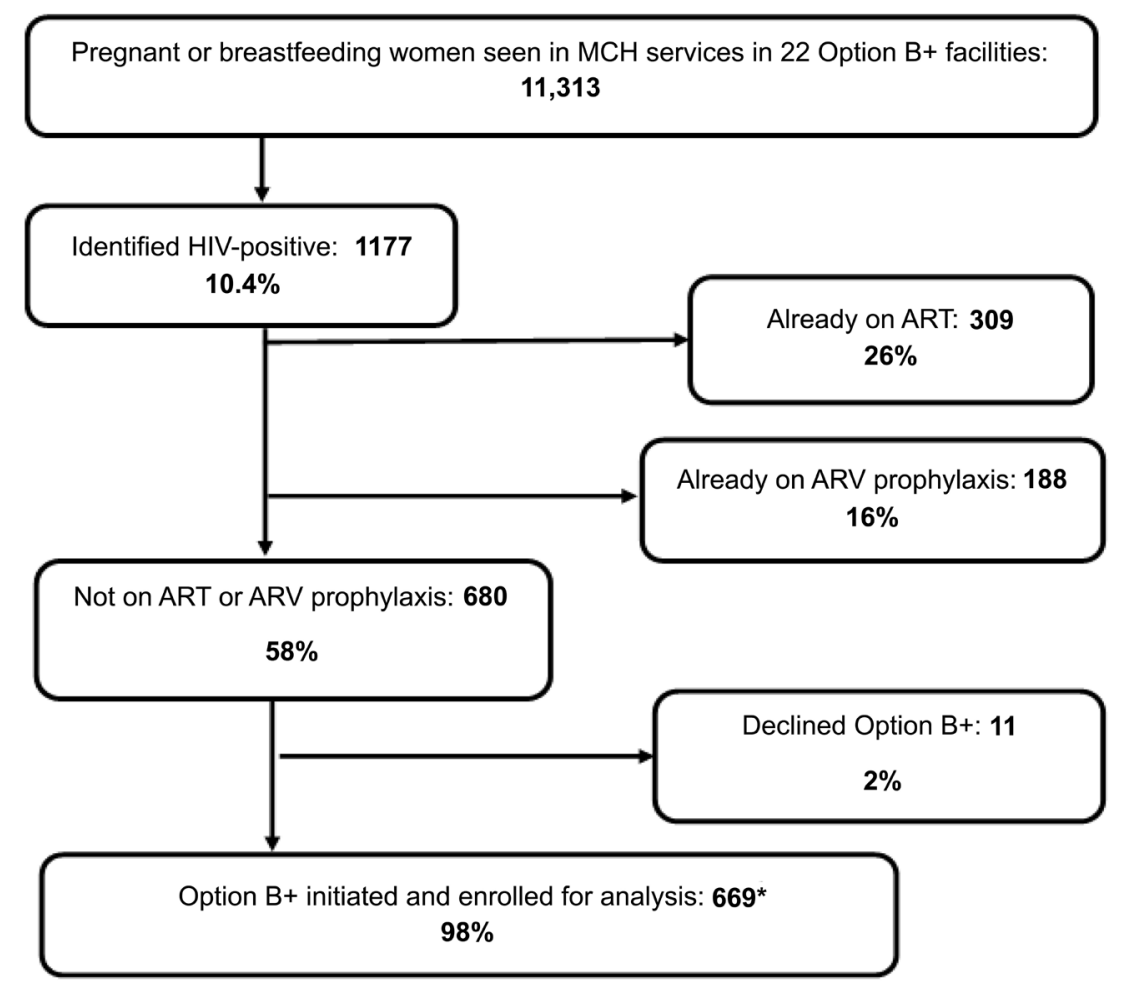

Figure 1. Enrolment flow chart of participants in Option B+ evaluation.

Table 1. Socio-demographic and clinical characteristics of ARV naïve pregnant and breastfeeding women enrolled for Option B+ evaluation.

\begin{tabular}{|c|c|c|}
\hline Variables & $\begin{array}{c}\text { Frequency } \\
(\mathrm{N}=669)\end{array}$ & $\%$ \\
\hline \multicolumn{3}{|l|}{ Age (years) } \\
\hline Median (IQR) & $27(24-31)$ & \\
\hline$<25$ & 252 & 37.7 \\
\hline $25-30$ & 217 & 32.4 \\
\hline$>30$ & 200 & 29.9 \\
\hline \multicolumn{3}{|l|}{ Marital Status } \\
\hline Single & 144 & 21.5 \\
\hline Married/Cohabiting & 522 & 78 \\
\hline Others (widow, divorced) & 3 & 0.5 \\
\hline \multicolumn{3}{|l|}{ Ownership of telephone } \\
\hline Yes & 608 & 90.9 \\
\hline No & 61 & 9.1 \\
\hline \multicolumn{3}{|l|}{ Preferred means of contact } \\
\hline Telephone call & 362 & 54.1 \\
\hline Home visit & 24 & 3.6 \\
\hline Both telephone and home & 270 & 40.4 \\
\hline Refused contact & 2 & 0.3 \\
\hline No means of contact & 3 & 0.5 \\
\hline
\end{tabular}




\section{Continued}

\begin{tabular}{|c|c|c|}
\hline Unknown & 8 & 1.2 \\
\hline \multicolumn{3}{|l|}{ Transport to site } \\
\hline$\leq 200 \mathrm{CFA}$ & 393 & 58.7 \\
\hline$>200 \mathrm{CFA}$ & 276 & 41.3 \\
\hline \multicolumn{3}{|l|}{ Health district } \\
\hline Bamenda & 431 & 64.4 \\
\hline Kumba & 238 & 35.6 \\
\hline \multicolumn{3}{|l|}{ Entry point } \\
\hline Antepartum (ANC/L \& D) & 626 & 93.6 \\
\hline Postpartum & 43 & 6.4 \\
\hline \multicolumn{3}{|l|}{ HIV status at enrolment } \\
\hline Known positive but not on ARVs & 215 & 32.1 \\
\hline Newly diagnosed & 454 & 67.9 \\
\hline \multicolumn{3}{|l|}{ WHO stage } \\
\hline I & 570 & 85.2 \\
\hline II & 72 & 10.8 \\
\hline III & 27 & 4 \\
\hline \multicolumn{3}{|l|}{ CD4 (cells $\left./ \mathrm{mm}^{3}\right)$ at enrolment } \\
\hline Median (IQR) & $364(225-496)$ & \\
\hline$\leq 350$ & 234 & 34.4 \\
\hline$>350$ & 257 & 38.4 \\
\hline Unknown & 178 & 26.6 \\
\hline
\end{tabular}

and breastfeeding women were included in the cohort at $0-6$ and $0-12$ months respectively. The average retention after ART initiation among participants who did not transfer out was 566 (90\%) (95\% CI, 87.85 - 92.61) at 0 - 6 months and 476 (79\%) (95\% CI, $75.20-81.88)$ at 0 - 12 months (Figure 2). Details of maternal outcome at $0-6$ and $0-12$ months after ART initiation are presented in Table 2.

At six months after ART initiation, 6 women (1\%) had died and 9 (1\%) had stopped ART. At twelve months after ART initiation, a total of 10 women (2\%) had died; 17 (3\%) had a still birth, and 15 (2\%) had a miscarriage. Of the 10 women who died, 2 died of HIV-related disease, 3 died of obstetrical complications, and the cause of death of five was unknown. An additional 12 women stopped their ART after six months for a total of 21 (3\%) women who stopped ART at 12 months (Table 2).

Table 3 shows the twelve-month distribution of LTFU among HIV-positive pregnant and breastfeeding women enrolled in the evaluation. Of the $98(15 \%)$ women who were LTFU at 12 months after ART initiation, 50 (51\%) never returned for their first ART refill (failure of ART initiation), and 48 (49\%) were LTFU after delivery. Spending less than 200 CFA (less than $\$ 0.5$ USD) to reach a 


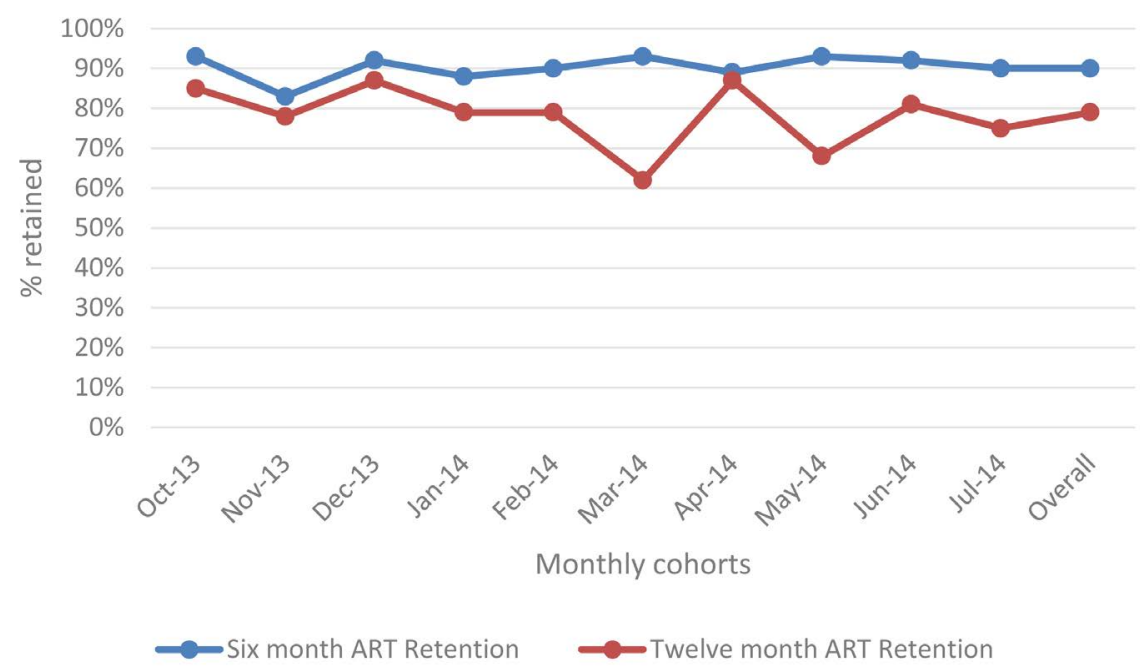

Figure 2. Retention for monthly cohorts of pregnant and breastfeeding HIV-positive women enrolled in Option B+ evaluation.

Table 2. Six and twelve month's maternal retention on ART among HIV-positive pregnant and breastfeeding women enrolled in Option B+ evaluation.

\begin{tabular}{|c|c|c|}
\hline \multirow{2}{*}{ Variables } & 0 - 6 months after ART & $0-12$ months after ART \\
\hline & \multicolumn{2}{|c|}{$\mathrm{N}=669$} \\
\hline Transfer out of study ${ }^{a}$ & Transfer out: $43(6 \%)$ & Transfer out: $64(10 \%)$ \\
\hline Because of miscarriage & $14(2 \%)$ & $15(2 \%)$ \\
\hline Because of still birth & $12(2 \%)$ & $17(3 \%)$ \\
\hline Because of relocation & $7(1 \%)$ & $14(2 \%)$ \\
\hline Because of infant death & $7(1 \%)$ & $11(2 \%)$ \\
\hline $\begin{array}{c}\text { Because of HIV DNA PCR positve } \\
\text { infant }^{\text {b }}\end{array}$ & $3(<1 \%)$ & $6(1 \%)$ \\
\hline Because of severe ART side effect & 0 & $1(<1 \%)$ \\
\hline Retention denominator ${ }^{c}$ & 626 & 605 \\
\hline Lost to follow up (LTFU) & LTFU: 45 (7\%) & LTFU: 98 (15\%) \\
\hline $\begin{array}{l}\text { Voluntarily discontinued ART } \\
\text { (voluntary withdrawal from study) }\end{array}$ & $9(1 \%)$ & $21(3 \%)$ \\
\hline Died & $6(1 \%)$ & $10(2 \%)$ \\
\hline Retained & 566 & 476 \\
\hline Retention $(95 \% \mathrm{CI})$ & $90.4 \%(87.9-92.6)$ & $76.7 \%(75.2-81.9)$ \\
\hline
\end{tabular}

a. Transferred out: defined as women who were referred and/or transferred to other facilities due to either miscarriage, stillbirth, infant death, or relocation, or a PCR positive infant; $b$. Infants with a positive HIV DNA PCR result were transferred to ART clinics for ART initiation but were also included in the MTCT rate for this study; $c$. Retention denominator was the number of clients on ART excluding the number who transferred out for each cohort at 6 and 12 months.

health facility (OR: 1.76, 95\% CI $1.14-2.71$ ) was identified as a factor that influenced LTFU. LTFU was not influenced by timing of enrollment, ownership of a telephone, HIV status at enrollment, newly diagnosed HIV-positive status at enrollment, or WHO clinical stage of disease (Table 3). 
Table 3. Twelve months distribution of LTFU among HIV-positive pregnant and breastfeeding women enrolled in Option B+ evaluation.

\begin{tabular}{|c|c|c|c|c|c|}
\hline Variables & $\begin{array}{l}\text { Frequency } \\
(N=669)\end{array}$ & $\begin{array}{l}\text { LTFU } \\
(\mathrm{n}=98)\end{array}$ & $\begin{array}{c}\% \\
(\mathrm{n} / \mathrm{Nx} 100)\end{array}$ & $\begin{array}{c}\text { OR } \\
(95 \% \mathrm{CI})\end{array}$ & $P$-value \\
\hline \multicolumn{6}{|l|}{ Age (years) } \\
\hline Median (IQR) & $27(24-31)$ & $26.5(23-29)$ & & & \\
\hline$<25$ & 252 & 42 & 16.7 & 1 & - \\
\hline $25-30$ & 217 & 38 & 17.5 & $1.06(0.69-1.72)$ & 0.81 \\
\hline$>30$ & 200 & 18 & 9 & $0.49(0.28-0.89)$ & 0.02 \\
\hline \multicolumn{6}{|l|}{ Marital Status } \\
\hline Single & 144 & 25 & 17.3 & 1 & - \\
\hline Married/Cohabiting & 522 & 73 & 13.9 & $0.77(0.47-1.27)$ & 0.31 \\
\hline $\begin{array}{l}\text { Others (widow, } \\
\text { divorced) }\end{array}$ & 3 & 0 & 0 & - & - \\
\hline \multicolumn{6}{|l|}{$\begin{array}{l}\text { Ownership of } \\
\text { telephone }\end{array}$} \\
\hline Yes & 608 & 84 & 13.8 & 1 & - \\
\hline No & 61 & 14 & 9.1 & $0.53(0.28-0.99)$ & 0.05 \\
\hline \multicolumn{6}{|l|}{$\begin{array}{l}\text { Preferred means of } \\
\text { contact }\end{array}$} \\
\hline Telephone call & 362 & 52 & 14.3 & 1 & - \\
\hline Home visit & 24 & 5 & 20.8 & $0.89(-7.33-28.61)$ & 0.35 \\
\hline $\begin{array}{c}\text { Both telephone and } \\
\text { home }\end{array}$ & 270 & 38 & 14 & $0(-5.60-5.82)$ & 1 \\
\hline Refused contact & 2 & 0 & 0 & $0.32(-70.26-18.01)$ & 0.57 \\
\hline No means of contact & 3 & 1 & 33.3 & $0.88(-13.44-76.53)$ & 0.35 \\
\hline Unknown & 8 & 2 & 25 & $0.77(-11.18-51.23)$ & 0.38 \\
\hline \multicolumn{6}{|l|}{ Transport to site } \\
\hline$\leq 200 \mathrm{CFA}$ & 393 & 46 & 11.7 & 1 & - \\
\hline$>200 \mathrm{CFA}$ & 276 & 52 & 18.9 & $1.76(1.14-2.71)$ & 0.01 \\
\hline \multicolumn{6}{|l|}{ Health district } \\
\hline Bamenda & 431 & 63 & 14.6 & 1 & - \\
\hline Kumba & 238 & 35 & 14.7 & $1.01(0.64-1.76)$ & 0.98 \\
\hline \multicolumn{6}{|l|}{ Entry point } \\
\hline $\begin{array}{l}\text { Ante partum } \\
\text { (ANC/L \& D) }\end{array}$ & 626 & 83 & 13.2 & 1 & - \\
\hline Post-partum & 43 & 15 & 34.9 & $0.94(0.39-2.29)$ & 0.89 \\
\hline \multicolumn{6}{|l|}{$\begin{array}{c}\text { HIV status at } \\
\text { enrolment }\end{array}$} \\
\hline $\begin{array}{c}\text { Known positive but } \\
\text { not on ARVs }\end{array}$ & 215 & 31 & 14.4 & 1 & - \\
\hline Newly diagnosed & 454 & 67 & 14.7 & $0.12(-5.23-6.66)$ & 0.73 \\
\hline \multicolumn{6}{|l|}{ WHO stage } \\
\hline I & 570 & 88 & 15.4 & 1 & - \\
\hline
\end{tabular}




\begin{tabular}{|c|c|c|c|c|c|}
\hline \multicolumn{6}{|l|}{ Continued } \\
\hline II & 72 & 7 & 9.7 & $0.59(0.26-1.33)$ & 0.2 \\
\hline III & 27 & 3 & 11.1 & $0.71(0.21-2.43)$ & 0.59 \\
\hline \multicolumn{6}{|c|}{$\begin{array}{c}\mathrm{CD} 4\left(\text { cells } / \mathrm{mm}^{3}\right) \text { at } \\
\text { enrolment }\end{array}$} \\
\hline Median (IQR) & $364(225-496)$ & & & & \\
\hline$\leq 350$ & 234 & 26 & 11.1 & 1 & - \\
\hline$>350$ & 257 & 32 & 12.5 & $0.12(-5.01-6.92)$ & 0.73 \\
\hline Unknown & 178 & - & - & - & - \\
\hline
\end{tabular}

\subsection{ARV and Cotrimoxazole Uptake, Retention and Mortality, among Enrolled HEIs}

Of the 669 women enrolled in the study, 120 women either died, had miscarriage, stillbirth or withdrew from care before delivery. The remaining 549 women gave birth to 568 HEIs; of these, 538 enrolled in the study. All 538 enrolled HEIs received postpartum NVP within 72 hours of birth, and 454 (84\%) received cotrimoxazole per national recommendations at 6 to 8 weeks of age. By 8 weeks of age, 498 (93\%) infants had results from an HIV DNA PCR test with 486 (97.6\%) tested negative and 12 (2.4\%) tested positive. All HIV-infected children were referred to ART clinics for immediate treatment. Details on enrollment and outcome of HEI are shown in Figure 3.

\section{Discussion}

Historically, PMTCT coverage and ART retention along the PMTCT cascade have been poor in Cameroon [4]. UNAIDS reported a 6 weeks MTCT rate of $5.2 \%$ in Cameroon, which is higher than the global eMTCT targets of less than $2 \%$ and $5 \%$ at 6 weeks and after cessation of breastfeeding respectively [13]. To achieve these targets, ART uptake and maternal retention on ART are essential. Early implementers of Option B+ have reported high ART uptake, yet many countries have struggled with maternal retention [14] [15]. In this evaluation, early retention at 6 months after ART initiation was high at $90 \%$ and then declined at 12 months after ART initiation to 79\%. The 12 months ART retention rate was high when compared to $60 \%$ from a nationwide survey of Cameroon's adult ART program in 2014 [16]. Among women in Option B+ programs, the 6 months retention rate in Cameroon was higher than the rate seen in Uganda and the Democratic Republic of Congo (DRC), while the 12 months retention was similar to data from Malawi Option B+ program [14] [17] [18]. To identify effective strategies to improve maternal retention, it was important to first understand which patients were at highest risk of LTFU. Similar to results from Malawi [17] and Ethiopia [15], this study found that clients with higher transport cost to the facility were twice as likely to become LTFU at 12 months compared to those with lower transport cost. To minimize LTFU after ART initiation and after delivery, we used appointment diaries and peer educators throughout the 
Pregnant or breastfeeding women enrolled in 22 health facilities: 669

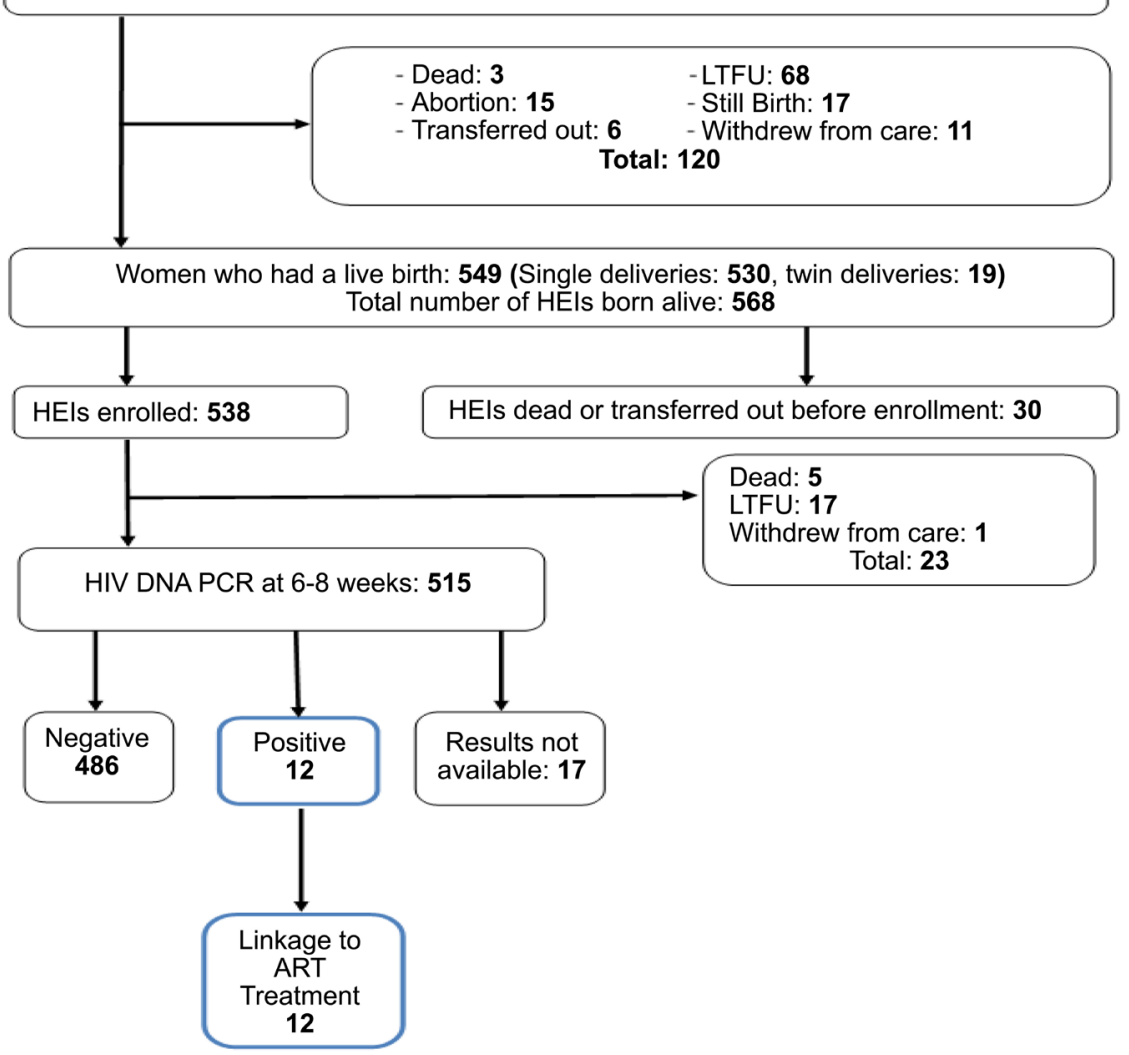

Figure 3. Enrollment and outcome of HIV exposed infants selected for Option B+ evaluation.

duration of the study, to closely monitor clients with missed appointments and to re-engage defaulters and patients with more than one month since the last ART refills. We did not experience high LTFU immediately after ART initiation and among women who initiated Option $\mathrm{B}+$ while breastfeeding. Compared to the Cameroon adult ART retention data, our study suggested that women involved in the Option $\mathrm{B}+$ program had a better 12 months ART retention, and this may be due to the interventions in place. However, this evaluation was limited to only 2 regions of Cameroon and was not nationwide as the Cameroon adult ART retention data.

In Malawi, 6 months retention data demonstrated that women initiating ART during pregnancy were five times more likely not to return to the clinic after they initiated ART compared to non-pregnant women (OR: 5.0, 95\% CI 4.2 6.1) and those who started Option B+ while breastfeeding were twice as likely to miss their first follow up visit compared to women who initiated during ANC [17].

During this evaluation, CD4 testing was used since the Cameroon national guidelines on management of HIV recommended CD4 testing at baseline and every 6 months while on ART. Despite cost subsidies and laboratory sample transportation systems to improve uptake of laboratory tests, we noted a drop in 
CD4 testing uptake greater than $50 \%$ at 6 months and almost $90 \%$ at 12 months after ART initiation. Reliance on ongoing CD4 monitoring for PMTCT Option $\mathrm{B}+$ for a test and start approach may not be feasible in the Cameroon setting.

Some of the challenges noted in this evaluation included systemic barriers (such as long waiting times for sample collection, stock out of reagents, frequent break down of equipment) and distance to testing site; these have been described in other countries as well [9] [10]. The results of this study stressed the importance of addressing programmatic challenges related to laboratory equipment and supply chain system of reagents and other commodities to ensure high uptake of routine laboratory testing.

In Cameroon, HIV is a large contributor to maternal mortality rates. A 2012 study attributed $10 \%$ of pregnancy-related deaths to HIV [6]. However, in this current study which took place in an Option B+ setting, a mortality rate of $2 \%$ was found among enrolled women at 12 months post-ART initiation; this is similar to $1.7 \%$ found in a study in Malawi [17] and 2.6\% found 6 months after initiation of Option B in the Cameroon DREAM study [19].

Rates of infant NVP uptake after birth, HIV DNA PCR testing at 6 to 8 weeks of life, and MTCT at 6 weeks captured by PCR were similar to those found in Malawi, where $95 \%$ of HEIs received ARV prophylaxis (NVP) after birth, $82 \%$ received a DNA PCR test at 6 to 8 weeks of life, and 2.6\% tested positive [20]. We put systems in place to track defaulters and improve retention along the PMTCT cascade and early infant diagnosis (EID) uptake using community workers.

The uptake of EID of $95.7 \%$ and the HIV transmission rate among infants tested at 8 weeks of $2.4 \%$ reported in this study are 3 times better than the 2014 overall country EID uptake of $24 \%$ and MTCT rate at 6 weeks of 7\%, identified shortly before Option B+ was implemented nationwide in Cameroon [21] The 2015 estimated uptake of EID at 8 weeks was 30\% while the HIV transmission rate among infants tested at 6 weeks was 5\% [22].

\section{Strengths and Limitations}

This evaluation follows a large prospective cohort of mother-infant pairs across 22 busy PMTCT sites predominantly in semi-urban and rural areas of Cameroon. The descriptive evaluation of the outcomes of mother-infant pairs was not designed as an experimental study that would allow causal inferences to be made. The selection strategy of participating health facilities and eligibility of participants may limit the generalizability of findings and the ability to be a model for a complete Option B+ policy. Because many HEIs were still breastfeeding at the time of the 6 weeks HIV DNA PCR testing, our results may underestimate final HIV rates in this cohort after cessation of breastfeeding. Additionally, we only know the HIV status of HEIs who were retained during the reporting period; HEIs who were not retained might have been more likely to become HIV infected or to have died. Final outcomes of enrolled HEIs are not included since many had not yet reached 18 months of age at the time of analysis. 
However, HEIs continued to be followed, and the HIV transmission rate among those tested at 24 months of age and/or after cessation of breastfeeding will be addressed in a subsequent analysis.

\section{Conclusion}

We describe the outcome evaluation of early implementation of PMTCT Option $\mathrm{B}+$ in Cameroon. Our data demonstrate that it is possible to achieve high 12 months ART retention rate among pregnant and breastfeeding women who start ART after they are diagnosed with HIV. In addition, reduction of MTCT was also achieved. Longer-term ART retention, infant mortality, and HIV transmission rate among HEIs after cessation of breastfeeding need further evaluation.

\section{Acknowledgements}

This publication was made possible by support from the President's Emergency Plan for AIDS Relief (PEPFAR) through cooperative agreement number 5U2GGH00352 from the Centers for Disease Control and Prevention (CDC), Division of Global AIDS Tuberculosis (DGHT). The findings and conclusions in this report are those of the author(s) and do not necessarily represent the official position of the Centers for Disease Control and Prevention. We would like to thank the University of North Carolina (UNC) for their assistance in the development of the Epi Info database used for data collection. We recognize colleagues at the Cameroon MOPH for their steadfast leadership and tireless work towards eliminating new HIV infections in children and keeping mothers healthy. We gratefully acknowledge Surbhi Modi, Team Lead for the Maternal and Infant HIV Team, Division of Global HIV/TB, Centers for Disease Control \& Prevention (CDC) and Nicholas Gaffga, Maternal and Infant HIV Team, Division of Global HIV/TB, Centers for Disease Control \& Prevention (CDC) for their technical review and contributions to the manuscript. We recognize management and staff of the 22 health institutions who participated in the evaluation for demonstrating exemplary technical leadership and collaboration. Finally, we wish to recognize the mothers living with HIV who participated in our study and were central to our collective successes in keeping the mother-infant pair healthy.

\section{Conflicts of Interest}

The authors declare no conflicts of interest regarding the publication of this paper.

\section{References}

[1] Cameroon: WHO statistical profile (2015). http://www.who.int/gho/countries/cmr.pdf

[2] UNAIDS (2016) Country Factsheets Cameroon. http://www.unaids.org/en/regionscountries/countries/cameroon 
[3] Cameroon Demographic and Health Survey and Multiple Indicators Cluster Survey (2011) (DHS-MICS). https://dhsprogram.com/pubs/pdf/FR260/FR260.pdf

[4] Rapport National de suivi de la Déclaration Politique sur le VIH/SIDA Cameroun. Global AIDS Response Progress (GARP).

http://www.unaids.org/sites/default/files/country/documents/CMR_narrative_repor t_2014.pdf

[5] WHO (2017) World Health Statistics. Monitoring Health for the Sustainable Developments Goals.

http://apps.who.int/iris/bitstream/handle/10665/255336/9789241565486eng.pdf

[6] WHO (2015) Trends in Maternal Mortality: 1990 to 2015. WHO, UNICEF, UNFPA, The World Bank Group and the United Nations Population Division.

http://apps.who.int/iris/bitstream/handle/10665/194254/9789241565141_eng.pdf

[7] Welty, T.K., Bulterys, M., Welty, E., Tih, P.M., Ndikintum, G., Nkuoh, G.N., Nkfusai, J., Kayita, J., Nkengasong, J.N. and Wilfert, C.M. (2005) Integrating Prevention of Mother-to-Child HIV Transmission Into Routine Antenatal Care. The Key to Program Expansion in Cameroon. Journal of Acquired Immune Deficiency Syndromes, 40, 486-493. https://doi.org/10.1097/01.qai.0000163196.36199.89

[8] Nkuoh, G.N., Meyer, D.J., Tih, P.M. and Nkfusai, J. (2010) Barriers to Men's Participation in Antenatal and Prevention of Mother-to-Child HIV Transmission Care in Cameroon. Journal of Midwifery \& Women's Health, 55, 363-369. https://doi.org/10.1016/j.jmwh.2010.02.009

[9] National AIDS Control Committee (2010) The Impact of HIV and AIDS in Cameroon through 2020 .

http://www.healthpolicyinitiative.com/Publications/Documents/1250_1_Cameroon EN_Singles_Reduced_acc.pdf

[10] Management Science for Health. Option B+ in Malawi: The origins and Implementation of a Global Health Innovation (2012).

https://www.msh.org/news-events/stories/option-b-in-malawi-the-origins-andimpl ementation-of-a-global-health-innovation

[11] Plan National d'élimination de la transmission mère enfant du VIH à l'horizon 2015 au Cameroun.

http://docplayer.fr/8196676-Plan-national-d-elimination-de-la-transmission-mereenfant-du-vih-a-l-horizon-2015-au-cameroun.html

[12] UNAIDS (2011) Countdown to Zero: Global Plan towards the Elimination of New HIV Infections among Children by 2015 and Keeping Their Mothers Alive, 2011-2015. Geneva, Switzerland.

http://files.unaids.org/en/media/unaids/contentassets/documents/unaidspublication /2011/20110609_JC2137_Global-Plan-Elimination-HIV-Children_en.pdf

[13] UNAIDS (2016) On the Fast-Track to an AIDS-Free Generation. http://www.unaids.org/sites/default/files/media_asset/GlobalPlan2016_en.pdf

[14] Kieffer, M. P., Mattingly, M., Giphart, A., van de Ven, R., Chouraya, C., Walakira, M., et al., the EGPAF Technical Directors Forum (2014) Lessons Learned From Early Implementation of Option B+: The Elizabeth Glaser Pediatric AIDS Foundation Experience in 11 African Countries. Journal of Acquired Immune Deficiency Syndromes, 67, S188-S194. https://doi.org/10.1097/QAI.0000000000000372

[15] Mitiku, I., Arefayne, M., Mesfin, Y. and Gizaw, M. (2016) Factors Associated with Loss to Follow-Up among Women in Option B+ PMTCT Programme in Northeast Ethiopia: A Retrospective Cohort Study. Journal of the International AIDS Society, 19, 20662. https://doi.org/10.7448/IAS.19.1.20662 
[16] Billong, S.C., Fokam, J., Penda, C.I., Amadou, S., Kob, D.S., Billong, E.-J., et al. (2016) Predictors of Poor Retention on Antiretroviral Therapy as a Major HIV Drug Resistance Early Warning Indicator in Cameroon: Results from a Nationwide Systematic Random Sampling. BMC Infectious Diseases, 16, 678.

https://doi.org/10.1186/s12879-016-1991-3

[17] Haas, A.D., Tenthani, L., Msukwa, M.T., Tal, K., Jahn, A., Gadabu, O.J., et al. (2016) Retention in Care during the First 3 Years of Antiretroviral Therapy for Women in Malawi's Option B+ Programme: An Observational Cohort Study. The Lancet. HIV, 3, 175-182. https://doi.org/10.1016/S2352-3018(16)00008-4

[18] Morbidity and Mortality Weekly Report (2013) Impact of an Innovative Approach to Prevent Mother-to-Child Transmission of HIV-Malawi, July 2011-September 2012. $M M W R, 62,148-151$.

https://www.ncbi.nlm.nih.gov/pmc/articles/PMC4604864/pdf/148-151.pdf

[19] Altan, A.M.D., Taafo, F., Fopa, F., Buonomo, E., Marazzi, M.C., Nielsen-Saines, K., et al. (2016) An Assessment of Option B Implementation for the Prevention of Mother to Child Transmission in Dschang, Cameroon: Results from the DREAM (Drug Resource Enhancement against AIDS and Malnutrition) Cohort. The Pan African Medical Journal, 23, 72.

[20] Kim, M.H., Ahmed, S., Hosseinipour, M.C., Giordano, T.P., Chiao, E.Y., Yu, X., et al. (2015) Implementation and Operational Research: The Impact of Option B+ on the Antenatal PMTCT Cascade in Lilongwe, Malawi. Journal of Acquired Immune Deficiency Syndromes, 68, 77-83. https://doi.org/10.1097/QAI.0000000000000517

[21] UNAIDS. A Progress Report on the Global Plan towards the Elimination of New HIV Infections among Children by 2015 and Keeping Their Mothers Alive. Geneva, Switzerland.

http://www.unaids.org/sites/default/files/media_asset/JC2774_2015ProgressReport_ GlobalPlan_en.pdf

[22] UNAIDS (2015) Progress Report on the Global Plan. Cameroon Factsheet. http://www.unaids.org/sites/default/files/media/documents/UNAIDS_GlobalplanC ountryfactsheet_cameroon_en.pdf 